\title{
La Educación en los Congresos Nacionales de Extensión Universitaria de Argentina
}

\author{
Education in the Argentineans National Congresses of University Extension
}

Marcelo Luis López*

\begin{abstract}
*Profesor y Licenciado en Ciencias de la Educación, posgraduado como Especialista en Docencia Superior (Facultad de Humanidades y Ciencias Sociales de la Universidad Nacional de Jujuy, FHYCS-UNJU). Se desempeña como docente/investigador concursado exclusivo de Práctica y Residencia en Ciencias de la Educación (FHYCS-UNJU) y Profesor de Didáctica General del Profesorado de la Escuela Superior de Música de Jujuy. Fue Coordinador de Extensión en la FHYCS-UNJU (2003-2006). Ha participado en numerosos proyectos de investigación, como investigador, co-irector y director, de los cuales derivan publicaciones de varios libros y artículos en revistas científicas indexadas nacionales y de otros países.
\end{abstract}

\section{RESUMEN}

En este artículo se abordan los resultados del análisis de la temática educativa en relación a ponencias de los primeros cuatro Congresos Nacionales de Extensión Universitaria de Argentina. En el problema investigado, es que existen líneas, temáticas y categorías de análisis para la praxis existencionista.se pueden inferir de la producción bibliográfica, la sistematización de experiencias, las reflexiones e investigaciones como aportes a la construcción del objeto extensión universitaria -y conceptos de su campo semántico y asociativo- a partir de las comunicaciones presentadas en estos eventos. Se utilizó como metodología la Teoría Fundamentada en los Datos y el Análisis de Redes Sociales. Los principales resultados y conclusiones hacen alusión a la identificación de objetos y sujetos sobre los que se construye la temática educativo-extensionista en los congresos, las implicancias de las redes bibliográficas y el papel que juega la categoría aprendizaje servicio.

Palabras clave: Extensión Universitaria - Educación Relación Teoría-Práctica - Congresos Nacionales - Argentina.

\section{ABSTRACT}

This article addresses the results of the analyzes of the educational issue in relation to the papers of the first four Congresses of University Extension in Argentina. The research problem is what thematic lines and categories of analysis for extension- ist praxis can be inferred from bibliographic production, the systematization of experiences, reflections and research as contributions to the construction of the object extension university from the communications presented in these Events. The Grounded Theory and Analysis of Social Networks was used as methodology. The main results and conclusions are: The identification of objects and subjects on which the educational-extensionist theme is constructed, the role of bibliographical networks and the theoretical category of service learning.

Keywords: University Extension - Education - Theory-Practice Relation - National Congresses - Argentina.

\section{INTRODUCCIÓN}

La Universidad argentina, en sus inicios, siguió un derrotero profesionalista según el modelo general de las universidades napoleónicas en que la educación del Estado asumía un rol estratégico -de allí la búsqueda de su carácter nacional- involucrada en la formación de las nuevas élites, la organización de la administración y la secularización de la cultura. La tendencia primaria fue basarse en la promoción de carreras liberales como Derecho y Medicina, más que en las ingenierías (como ocurriría en otros países como Japón) lo que no impidió que más adelante se desarrollaran líneas con importantes logros en la investigación acercándola a un modelo humboldtiano (Vasen, 2013; Brunner, 2014). La irrupción de la extensión universitaria en el siglo XIX (Palacios Morini, 1908) y su afianzamiento en Latinoamérica, durante el siglo siguiente, probablemente se estableció a partir del trasfondo ético (Carlevaro, 2010) que la relaciona con principios y valores vinculados a la promoción social, algo especialmente significativo en países como la Argentina, que poseen riqueza pero, a la vez, desigualdad en su distribución.

En la República Argentina, la plataforma de lanzamiento de la extensión universitaria tuvo, en principio, a las universidades públicas estatales de La Plata, Córdoba, Buenos Aires y el Litoral, siendo "el llamado" de la Reforma Universitaria de 
Perspectivas Docentes 64

La Educación en los Congresos Nacionales de Extensión Universitaria de Argentina

Education in the Argentineans National Congresses of University Extension

Marcelo Luis López

Córdoba de 1918 la que la instala fuertemente persiguiendo el objetivo de superar el aislamiento, situándola como una herramienta para este cometido y una función que cambió la fisonomía del panorama de la educación superior. A fines del siglo XX las políticas neoliberales, a nivel mundial y en muchos países de América del Sur, produjeron la retracción del Estado en temas sociales y la necesidad de buscar nuevos actores o potenciar otros que ya estaban presentes -como la Universidad- para que ocuparan este lugar según queda plasmado en la Primera Conferencia Mundial de Educación Superior de 1998, y subsiguientes, lo que generó mucha actividad extensionista y la necesidad de socializar experiencias en una gran multiplicidad de eventos (López, 2012).

Es en este marco que, a partir de 1997, se desarrollan los Congresos Nacionales de Extensión Universitaria de Argentina. En base a estas cuestiones se desarrolló una tesis doctoral recortada a los primeros cuatro congresos Nacionales de Extensión Universitaria de Argentina, dando continuidad a una línea de investigación ya iniciada sobre extensión universitaria.

El problema planteado es: Qué líneas temáticas y categorías de análisis para la praxis extensionista se pueden inferir de la producción bibliográfica, la sistematización de experiencias, las reflexiones e investigaciones como aportes a la construcción del objeto extensión universitaria -y conceptos de su campo semántico y asociativo- a partir de las comunicaciones presentadas en estos eventos. La relevancia del asunto viene dado por la falta de teorización e investigación en una función como la extensión universitaria que, si bien aparece muy presente en el discurso universitario, se desarrolla muy pragmáticamente. A través de los 14 años que abarcaron estos primeros cuatro congresos se presentaron una gran cantidad de ponencias que se pudieron reagrupar en una serie de temas (ver tabla I). Este artículo, se ocupa, particularmente, de la temática educativa y dentro de ella dos categorías emergentes que son: los aportes de las redes bibliográficas -sobre educación- a la construcción del objeto extensión universitaria y el papel del organizador aprendizaje servicio.

La temática educación reagrupa, por un lado, aquellos trabajos vinculados a los procesos de transferencia de cultura -con diferentes grados de institucionalización- que, desde la perspectiva reproductivista, considera a la educación como fundamental para la reproducción fenomenológica de la sociedad, la homogeneización de una conciencia ciudadana y la diversificación para el mundo del trabajo (Durkheim, 1922). Por otro lado, está el punto de vista de los Reproductivistas Críticos, que refiere a la educación más como procesos de transferencia de ideología en que la educación, la acción pedagógica y la institución escolar aparecen como factores determinantes de la imposición de significados (violencia simbólica) que se realiza desde un arbitrario cultural dominante, disimulado para interiorizar relaciones de mando-subordinación funcionales al mundo del trabajo donde, además, se distribuyen conocimientos de manera selectiva y diferencial para lograr el mantenimiento del statu-quo con la consecuencia aparejada de la injusticia y la desigualdad social (Bowles y Gintis, 1970; Bourdieu y Passeron, 1979).

TABLA I. Temáticas desarrolladas en los primeros cuatro Congresos Nacionales de Extensión Universitaria de Argentina

\begin{tabular}{lc}
\hline \multicolumn{1}{c|}{ TEMÁTICAS } & Cant. de ponencias \\
\hline SALUD & 261 \\
EDUCACIÓN & 181 \\
EXTENSIÓN UNIVERSITARIA & 162 \\
PRODUCCIÓN PRIMARIA & 97 \\
DERECHOS (Derecho/Cs. Jurídicas, contextos de encierro, DD.HH. Género). & 54 \\
COMUNICACIÓN & 49 \\
ECONOMÍA & 48 \\
ARQUITECTURA/URBANISMO & 45 \\
HUMANIDADES/ARTE/CULTURA & 37 \\
CIENCIAS SOCIALES & 25 \\
INGENIERÍA & 25 \\
CIENCIAS EXACTAS Y/O NATURALES & 23 \\
TRABAJO SOCIAL / SERVICIO SOCIAL & 22
\end{tabular}


Perspectivas Docentes 64

La Educación en los Congresos Nacionales de Extensión Universitaria de Argentina

Education in the Argentineans National Congresses of University Extension

Marcelo Luis López

$\begin{array}{lr}\text { INFORMÁTICA } & 18 \\ \text { POLÍTICA (Política/Ciencias Políticas/ Políticas Públicas/Sociales) } & 17 \\ \text { TURISMO } & 15 \\ \text { MEDIOAMBIENTE } & 14 \\ \text { SOCIOLOGÍA } & 14 \\ \text { PATRIMONIO } & 12 \\ \text { ANTROPOLOGÍA } & 10 \\ \text { DESARROLLO / LOCAL / SUSTENTABLE } & 9 \\ \text { PUEBLOS ORIGINARIOS } & 9 \\ \text { CIENCIAY TECNOLOGÍA } & 8 \\ \text { ESTUDIOS LABORALES } & 7 \\ \text { VETERINARIA } & 6 \\ \text { PRODUCCIÓN EN GENERAL } & 3 \\ \text { ESTADÍSTICA } & 2 \\ \text { INSTITUCIÓN POLICIAL } & 2 \\ \text { OTROS } & 10 \\ \text { SIN CONSIGNAR } & 72\end{array}$

\section{METODOLOGÍA}

Se trata de un diseño no experimental, de tipo combinado cualitativo-cuantitativo, sustentado en un enfoque epistemológico interpretativo. Las fuentes de información fueron documentales conformadas por los libros de resúmenes y ponencias de los primeros cuatro Congresos Nacionales de Extensión Universitaria de Argentina (Cuyo, 1997; Mar del Plata, 2006; Santa fe, 2009; Cuyo 2010). Se trabajó desde la perspectiva metodológica de la teoría fundamentada en los datos (TFD) (Gaete Quezada, 2014) incluyendo, además, la aplicación de la lógica de Análisis de Redes Sociales (ARS) (Avila Toscano, 2012) con los programas de Análisis de Redes Sociales (UCINET 6.0) y NetDraw para vincular ponencias que contuvieran bibliografía sobre EU como uno de los criterios para iniciar el muestreo teórico y determinar las unidades muestrales conducentes al análisis cualitativo de un universo integrado por $n=1257$ ponencias (a cada una de las cuales se le asignó un número único de identificación). Para el muestreo teórico de la TFD se aplicó el ARS bajo el criterio rector de definir unidades muestrales iniciales (ponencias) con potencialidad para construir teoría en relación al problema de investigación. Luego de las lecturas preliminares se determinó trabajar con las producciones que contuvieran sustento bibliográfico sobre EU.
Ello sin embargo excluía al congreso de Cuyo 97 -que no contaba con libro de trabajos completos en el que se consignara la bibliografía de cada ponencia- aspecto que determinó leer previamente todos los resúmenes para detectar producciones que tuvieran algún rasgo particular en relación a otros aportes clave para luego ir a los trabajos completos. Aquí no se desarrollan las etapas conclusivas de la TFD (comparación de homogeneidades y heterogeneidades, diagrama lógico y el paradigma de codificación) puesto que se trata de un avance parcial referido sólo a la temática educativa.

\section{RESULTADOS}

Se identificaron 181 ponencias referidas a Educación que constituyeron un $14 \%$ del total de los trabajos presentados a través de los cuatro congresos, o sea el segundo tema en volumen de aportes. Si se toma como indicador de interés por la temática la cantidad de ponencias, se puede concluir que en el caso de Educación hubo un aumento sostenido que se expresó en un incremento en cada evento, según lo ilustra la tabla II: 
Perspectivas Docentes 64

La Educación en los Congresos Nacionales de Extensión Universitaria de Argentina

Education in the Argentineans National Congresses of University Extension

Marcelo Luis López

TABLA II. Ponencias respecto a la temática de Educación en los primeros Congresos Nacionales de Extensión Universitaria (1997-2010)

\begin{tabular}{|l|l|l|}
\hline \multicolumn{1}{|c|}{$\underline{\text { Congreso }}$} & \multicolumn{1}{|c|}{ Ponencias } & \multicolumn{1}{c|}{$\frac{\text { Ponencias sobre }}{\text { Educación }}$} \\
\hline Cuyo (1997) & 259 & 24 \\
\hline Mar del Plata (2006) & 217 & 36 \\
\hline Santa Fe (2009) & 387 & 54 \\
\hline Cuyo (2010) & 394 & 67 \\
\hline Total & $\mathbf{1 2 5 7}$ & $\mathbf{1 8 1}$ \\
\hline
\end{tabular}

Los sujetos y los objetos de la extensión universitaria de las ponencias, en la temática de educación, a través de los 14 años en que se extendieron estos primeros 4 congresos, son diversos. Respecto a los primeros tuvieron preponderancia respectivamente los docentes, estudiantes e instituciones escolares; luego grupos etarios donde parece haber una mayor preocupación por jóvenes y adolescentes (en segundo término, por niños y adultos/adultos mayores). Si bien el abordaje de estos sujetos sigue un derrotero fluctuante en términos de ponencias presentadas en cada congreso, las instituciones escolares parecen mostrar una atención creciente. En relación con los objetos de la extensión universitaria, en la temática educativa se destacan los procesos de alfabetización, la articulación entre la universidad y el nivel medio, la problemática de integración, de manera incipiente las tecnologías de la información y comunicación (TIC) -en los últimos dos congresos- y la enseñanza/aprendizaje sobre temas específicos (educación artística, física, matemática, medioambiente y otros).

La cuestión de la articulación entre la universidad y el nivel medio es un tema que cobra impulso en el marco de los Consejos de Planificación Regional de la Educación Superior (CPRES) creados por el art. 10 de la Ley de Educación Superior $(24.521 / 95)$ y los programas de la Secretaría de Políticas Universitarias.

Desde el punto de vista de algunos organizadores propios del sistema educativo como los niveles y modalidades, cuando se examinan las ponencias se encuentra que la preminencia sigue el siguiente orden: El nivel medio $13 \%$ de las exposiciones, luego el nivel superior $9 \%$, nivel primario $8 \%$, adultos $5 \%$, discapacidad y educación no formal $1 \%$ (cada uno); el resto de las ponencias presenta una gran dispersión inespecífica.
- La red bibliográfica sobre educación se compone de 1165 referencias que se distribuyeron entre 157 ponencias (el Congreso de Cuyo de 1997 no posee libro de trabajos completos por lo que se excluye del análisis bibliográfico). El grafo de la figura 1 ilustra la situación de la red sobre este tema a partir del cual se puede inferir que:

- De 157 ponencias, 66 (42\%) del total, comparten bibliografía.

- $\quad$ La red se compone de una red central, 7 islas y 91 ponencias aisladas (no vinculadas o que no comparten bibliografía).

- La densidad de la red es $=0,0080$ (entre dos a tres veces mayor a lo que ocurre por ejemplo con la temática de salud que es una de las más prominentes).

- Las ponencias con mayor centralidad e intermediación son: 759 (nodos de grado 12); 884 (grado 10); 1025, 586 y 370 (grado 8), 1061 y 922 (grado 7), 994, 679 y 602 (grado 6). Lo que es destacable es que las ponencias con mayor centralidad e intermediación (exceptuando la 1061 y 884) contienen y comparten, con otras ponencias, bibliografía vinculada a extensión universitaria, produciendo un núcleo de autores y exposiciones que pueden estar significando potencialmente el despegue hacia un núcleo de representaciones comunes sobre extensión universitaria en y desde la temática educativa, hacia el resto de las temáticas extensionistas.

- Se detectaron dos camarillas que corresponden a grupos de ponencias de un mismo autor o grupo de autores que comparten la misma base bibliográfica: En Santa Fe (2009) ponencias 548 y 549; Luego las ponencias 738,764 y 1255 , de las cuales, las primeras dos fueron presentadas en Santa Fe (2009) y la última en Cuyo (2010). 
Perspectivas Docentes 64

La Educación en los Congresos Nacionales de Extensión Universitaria de Argentina

Education in the Argentineans National Congresses of University Extension

Marcelo Luis López

FIGURA I. Red bibliográfica de Educación de los Congresos Nacionales de Extensión Universitaria (1997-2010) en el que se han despejado las islas y red central de ponencias que comparten bibliografía.

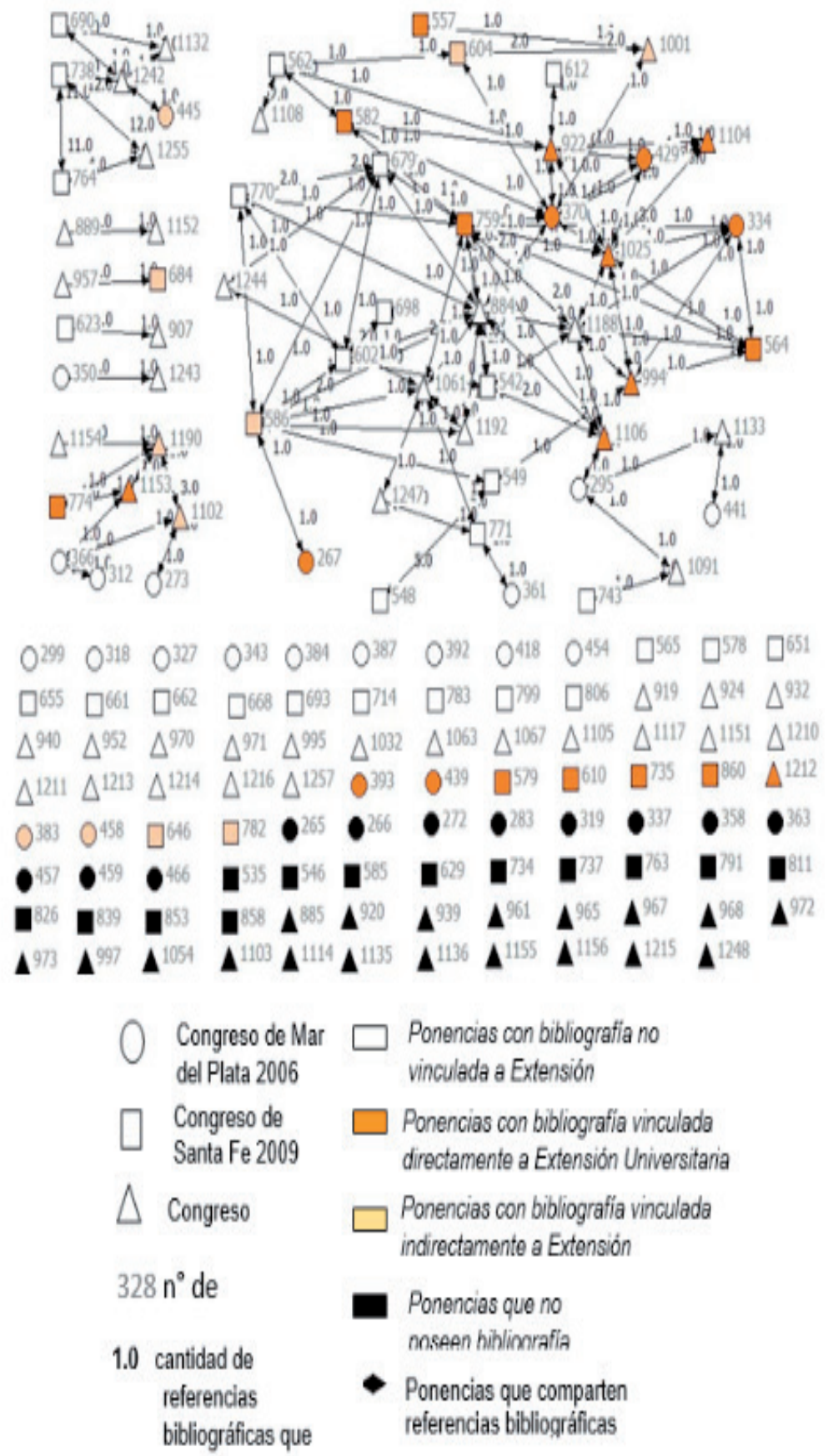

Por otra parte se puede derivar del grafo el siguiente cuadro distributivo de ponencias reagrupadas por congreso y en relación a los criterios de clasificación (Tabla III): 
Perspectivas Docentes 64

La Educación en los Congresos Nacionales de Extensión Universitaria de Argentina

Education in the Argentineans National Congresses of University Extension

Marcelo Luis López

TABLA III. Composición de la bibliografía para el área Educación en los Congresos Nacionales de Extensión Universitaria (19972010) en relación al objeto Extensión Universitaria.

\begin{tabular}{|l|l|l|l|l|}
\cline { 2 - 5 } \multicolumn{1}{c|}{} & $\begin{array}{l}\text { Mar del } \\
\text { Plata 2006 }\end{array}$ & $\begin{array}{l}\text { Santa Fe } \\
\mathbf{2 0 0 9}\end{array}$ & Cuyo 2010 & Total \\
\hline $\begin{array}{l}\text { Ponencias con bibliografía no vinculada a Extensión Universitaria o su campo } \\
\text { semántico y/o asociativo. }\end{array}$ & 16 & 27 & 38 & 81 \\
\hline $\begin{array}{l}\text { Ponencias con bibliografía vinculada directamente a Extensión universitaria o su } \\
\text { campo semántico y/o asociativo. }\end{array}$ & 6 & 9 & 7 & 22 \\
\hline $\begin{array}{l}\text { Ponencias con bibliografía vinculada indirectamente a Extensión universitaria o su } \\
\text { campo semántico y/o asociativo. }\end{array}$ & 3 & 5 & 3 & 11 \\
\hline Ponencias sin bibliografía. & 11 & 13 & 19 & 43 \\
\hline
\end{tabular}

El uso de la bibliografía en los trabajos, según lo que muestra la tabla 3 evidencia, a través de los congresos, crecimiento tanto de las ponencias sin bibliografía como de aquellas con bibliografía vinculada a la especialidad con escasez de material vinculado a extensión universitaria.

Desde un punto de vista más cualitativo, respecto a las referencias bibliográficas, la particularidad es la recurrencia a autores y obras que orientan teórica y filosóficamente acerca de lo que es y lo que debería ser la extensión universitaria. En este caso, la categoría sería: textos que orientan la concepción extensionista. Se destacan dos obras: "¿Extensión o comunicación? La concientización en el medio rural" de Paulo Freire (1973) y "Extensión universitaria y educación popular" de Silvia Brusilovsky, (1999). Respecto a la primera, Paulo Freire aborda alfl la problemática de la extensión en la ruralidad, y los campesinos alfabetizandos -el sujeto fundamental de su obrapor lo menos en la primera etapa. El organizador extensión aparece aquí como un elemento discursivo en el campo de la ruralidad pero que se conceptualiza críticamente como distinto al proceso de una comunicación emancipadora al que debería aspirarse. Se entiende a unos (profesionales y técnicos de la ruralidad) que detentan el conocimiento y que extienden este saber sobre otros que actúan como supuestos beneficiarios pasivos de esta acción. Esta asimetría de poder entre los representantes de opresores y oprimidos (también ubicados en su dialéctica como potenciales opresores en germen) son polos que otros autores (Bourdieu y Passeron, 1979) identificarán como la diferencia esencial entre un proceso comunicativo y uno pedagógico, desde el punto de vista de la Pedagogía Crítica y los Reproductivistas Críticos. De este modo, Freire deja planteada una crítica esencial al concepto de extensión y las prácticas que derivan de éste, al tiempo que propone repensarlo.
Por otra parte, Silvia Brusilovsky en la obra precitada recupera, a través de una investigación, una experiencia en la línea de la educación popular desarrollada en el Departamento de Extensión Universitaria de la UBA entre 1956 y 1966 que se presenta como un modelo inspirador alternativo a las políticas neoliberales de los 90 (cuando escribe este libro). Ambos trabajos generan una conceptualización de la Extensión desde una perspectiva socio-crítica evidenciando las potencialidades de la extensión universitaria en el ámbito de la educación popular y el circuito no formal.

Aunque muchos de los sujetos y objetos atendidos en las ponencias sobre educación de los congresos se concentran en determinados nichos, sin embargo, algunos aparecen fuertemente transversalizados a muchas temáticas constituyendo un rasgo de saturación de las categorías (tal es el caso de todo lo asociado a los contextos de privación de la libertad como la problemática de internos, liberados e instituciones penitenciarias, la integración de funciones docencia-investigación-extensión y la problemática medioambiental). En la temática educativa esto se evidencia, particularmente, con el organizador aprendizaje-servicio. En las ponencias predomina una conceptualización referida a un conjunto de prácticas que involucran la participación ciudadana de los estudiantes en experiencias solidarias orientadas a dar respuestas a necesidades de la comunidad, lo que supone una mejor comprensión de los contenidos curriculares que, en algunos casos, quedan subordinados a un aprendizaje por proyectos que requiere abordajes interdisciplinarios (Cecci, 2006; García \& Justicia 2007; Tapia, 2010). El organizador aprendizaje-servicio emerge con bastante fuerza y consenso en varios trabajos, y se instala especialmente en los dos últimos congresos. Por ser el "aprendizaje" un objeto subsidiario del campo educativo-educacional corresponde ubicarlo en esta 
Perspectivas Docentes 64

La Educación en los Congresos Nacionales de Extensión Universitaria de Argentina

Education in the Argentineans National Congresses of University Extension

Marcelo Luis López

temática aunque como un eje transversal ya que aparece vinculado a ponencias de diversas temáticas, además de la educativa: Salud (ponencias 587, 980 y 1141), Arquitectura/ Urbanismo (ponencias 603 y 989), Veterinaria (ponencias 732, 835 y 880), Extensión Universitaria (ponencias 348 y 890), Trabajo Social (ponencia 945), Economía (ponencia 959), Ciencias Sociales (ponencia 1118).

\section{DISCUSIÓN Y CONCLUSIONES}

Respecto a la red bibliográfica de las ponencias sobre educación, si se compara con el resto de las temáticas que arrojaron resultados (figura 2), se puede observar que, aun con escasez de bibliografía vinculada a extensión universitaria, la educación es unárea que muestra una de las redes bibliográficas más profusas. Los expositores utilizan y reutilizan, a través de los congresos, un conjunto de textos que actúa como núcleo orientador en relación a la extensión universitaria. Además de P. Freire y Silvia Brusilovsky, desde el plano educativo, cuyas obras son mencionadas en 187 y 22 oportunidades respectivamente, otros autores y trabajos que aparecen con menor frecuencia son los de Risieri Frondizi, François Vallaeys (responsabilidad social universitaria) y Humberto Tommasino (prácticas integrales), sin dejar de mencionar los aportes de José Porras (emprendedurismo y desarrollo local), Gustavo Menéndez de la Universidad Nacional del Litoral, María Raquel Coscarelli de la Universidad Nacional de la Plata y María Inés Peralta de la Universidad Nacional de Córdoba. Se puede decir que, si los congresos constituyen mínimamente un reflejo de lo que acontece en materia de extensión universitaria en la Argentina y/o la región, este grupo de autores, en su conjunto y en ese orden, son los que probablemente más han influido desde el plano discursivo respecto a cómo organizan intelectualmente la extensión universitaria los extensionistas de la Argentina entre 2006 y 2010, incluyendo la temática educativa. Esto señala a esta temática como una de las posibles y principales plataformas de lanzamiento desde el que se puede estar organizando conceptualmente el despegue de la extensión universitaria en la región. 
Perspectivas Docentes 64

La Educación en los Congresos Nacionales de Extensión Universitaria de Argentina

Education in the Argentineans National Congresses of University Extension

Marcelo Luis López

FIGURA II. Figura 2. Comparación de los grafos que muestran las redes bibliográficas de las distintas temáticas de los congresos.
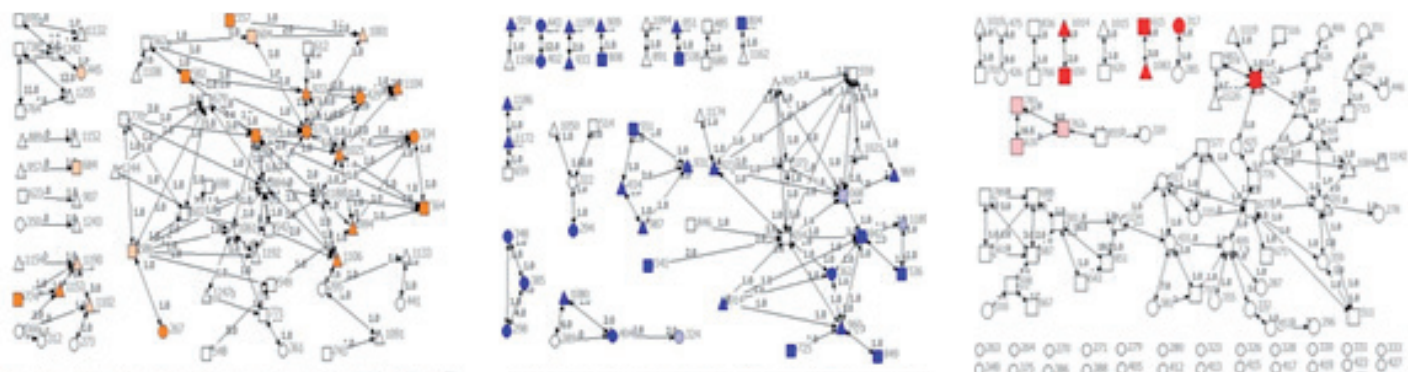

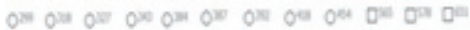

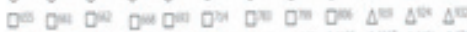

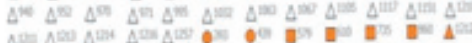

$0_{0}=a^{2}$

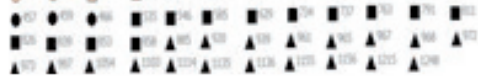

EDUCACION

EXTENSION LNIUERSITARIA
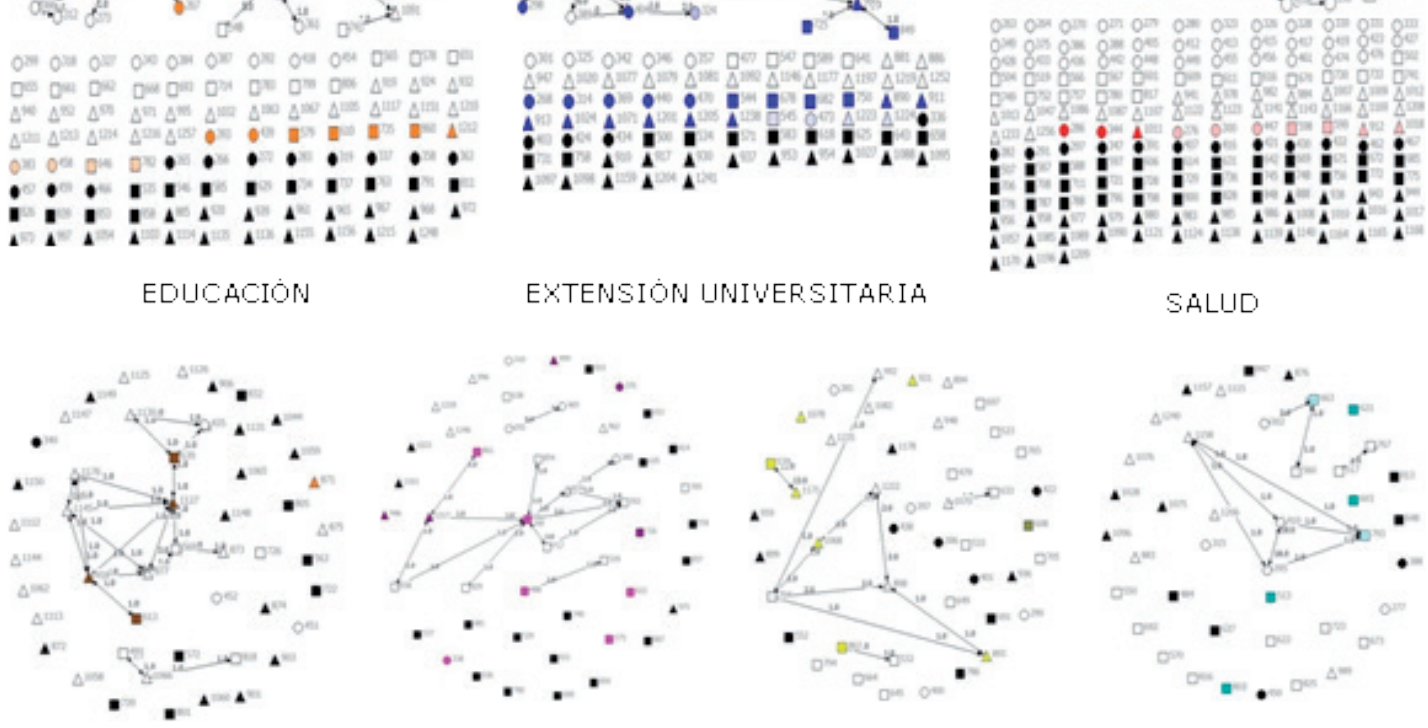

DERECHOS

COMUNICACION

ECONOMIÁ

ARQUITECTURÁ

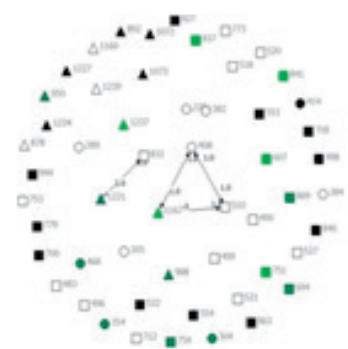

PRODLICCIÓN PRIMÁRIÁ

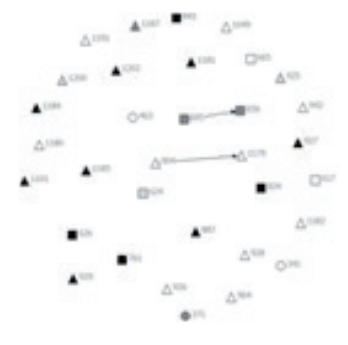

HUMANIDADES

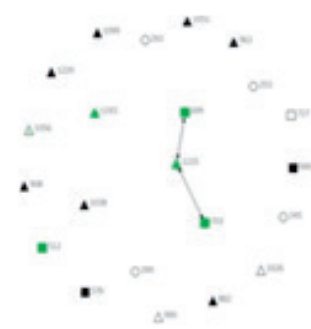

INGENIERÍA

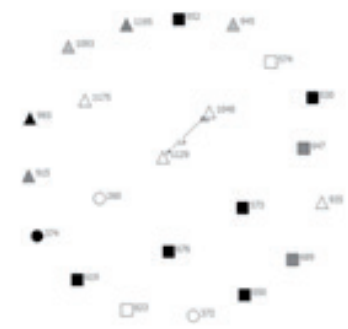

TRABAJO SOCIAL 
Perspectivas Docentes 64

La Educación en los Congresos Nacionales de Extensión Universitaria de Argentina

Education in the Argentineans National Congresses of University Extension

Marcelo Luis López

Respecto al aprendizaje servicio, aunque a menudo, en las ponencias y en la bibliografía, se lo presenta desde una perspectiva socio-crítica y socio-histórica vinculándolo con Freire y con Vigotsky (García García y Cotrina García, 2015), aparece más conectado a enfoques sistémicos que ignoran el obstáculo que representa la situación de clase ad-origen de los sujetos intervinientes en estos procesos para una transformación profunda como a la que parece aspirar el aprendizaje servicio, no quedando claro, entonces, cuáles son sus bases psicopedagógicas. Si bien hay trabajos que abordan con mayor profundidad teórica costados del aprendizaje servicio como facilitador de redescripciones representacionales implicadas en el cambio conceptual desde modelos cálidos, que incluyen aspectos motivacionales-emocionales, respecto de modelos fríos basados primordialmente en estrategias cognitivas (Ruiz-Danegger, 2009), sin embargo, no es la perspectiva que predomina en los congresos, que poseen abordajes más superficiales. De todos modos, no deja de ser una realidad insoslayable el fuerte papel orientador que ha tenido el organizador aprendizaje-servicio para los extensionistas durante el período abordado.

Finalmente, se puede agregar que, en los primeros cuatro Congresos Nacionales de Extensión Universitaria de Argentina, los sujetos y objetos más recortados en materia de educación fueron los jóvenes escolarizados en el nivel medio ayudando a interpretar y construir esta problemática desde el punto de vista de la relación sociedad-universidad.

\section{REFERENCIAS}

Ávila Toscano, J. H. (2012). Redes sociales y análisis de redes. Aplicaciones en el contexto comunitario y virtual. BarranquillaColombia: Azul y Violeta Editores Ltda.

Bourdieu, P.; Passeron, J. C. (1998). La reproducción. (1ed. 1979). México: Fontamara.

Bowles, S.; Gintis, H. (1986). La Instrucción Escolar en la América Capitalista. (10 ed. 1970). México: Siglo XXI Editores.

Brunner, J. J. (2014) La idea de la universidad pública en américa latina: narraciones en escenarios divergentes. UNED Educación XX1 17(2), 17-34. DOI: 10.5944/educxx1.17.2.11477
Brusilovsky, S. L. (1999). Extensión universitaria y educación popular: experiencias realizadas, debates pendientes. Buenos Aires: Eudeba.

Carlevaro, P. V. (2010). Reflexiones sobre la práctica educativa universitaria y la formación ética. En del Huerto Nari, María; Ibáñez, Ana (Coordinadoras). Formación Ética en la Universidad. Uruguay: Universidad de la República.

Cecci, N. (2006) Aprendizaje servicio en educación superior, la experiencia Latinoamericana en Seminario Internacional de Responsabilidad Social Universitaria: Aprendizaje Servicio. Caracas.

Durkheim, É. (1996) Educación y Sociología. (10 Ed. 1922). Barcelona: Ediciones Península.

Freire, P. (1973) ¿Extensión o Comunicación? La concientización en el medio Rural. Buenos Aires: Siglo XXI.

Gaete Quezada, R. (2014) Reflexiones sobre las bases y procedimientos de la Teoría Fundamentada. Ciencia, Docencia y Tecnología, 1(48), mayo, 149 - 172. Recuperado de http://www.revistacdyt.uner.edu.ar/spanish/cdt_48/ documentos/005cdt_48.pdf

García García, M; Cotrina García M. J. (2015). Formación inicial del profesorado: de las prácticas educativas críticas a la institucionalización curricular. Revista de currículum y formación del profesorado, 1(19), 8-25. Recuperado de https:// recyt.fecyt.es/index.php/profesorado/article/view/41019/23309

García y Justicia, J. (2007) El aprendizaje-servicio en la universidad, RSU, voluntariado y aprendizaje- servicio en la Educación Superior, Buenos Aires, Actas del $10^{\circ}$ Seminario Internacional Aprendizaje y Servicio Solidario.

Libro del IV Congreso Nacional e Extensión Universitaria y IX Jornadas Nacionales de Extensión Universitaria "Compromiso social y calidad educativa: desafíos de la Extensión". (2010). Cuyo-Mendoza: EDIUNC.

Libro de resúmenes (impreso) y de ponencias (CD) del III Congreso Nacional de Extensión Universitaria "La Integración, Extensión, Docencia e Investigación. Desafíos para el 
Perspectivas Docentes 64

La Educación en los Congresos Nacionales de Extensión Universitaria de Argentina

Education in the Argentineans National Congresses of University Extension

Marcelo Luis López

Desarrollo Social" (2009). Santa Fe-Argentina: Universidad Nacional del Litoral.

Libro de resúmenes del I Congreso Nacional de Extensión de la Educación Superior. II Encuentro Latinoamericano de Extensión Universitaria (1997). Cuyo-Mendoza-Argentina: EDIUNC.

Libro del II Congreso de Extensión Universitaria: un intercambio con la comunidad. (2006). Mar del Plata-Argentina: Ediciones Suárez.

López, M. L. (2012). Extensión universitaria situación actual y aportes metodológicos. Jujuy-Argentina: EdiUNJu.

Palacios Morini, L. (2002). Universidades populares. [Versión digital HTLM] (Edición original de Sempere y Compañía Editores 1908) Gijón-España: Fundación Municipal de Cultura. Recuperado de http://www.filosofia.org/aut/lpm/index.htm
Ruiz-Danegger, C. (2009). Aportes para la teorización sobre el aprendizaje-servicio en la universidad. VI Encuentro Nacional y III Latinoamericano La Universidad como Objeto de Investigación, Universidad Nacional de Córdoba, Córdoba, Argentina. Recuperado de https://www.academia. edu/15768840/Aportes_para_la_teorizaci\%C3\%B3n_sobre _ el_aprendizaje-servicio_en_la_universidad.

Tapia, M. N. (2010). La propuesta pedagógica del "AprendizajeServicio. TZHOECOEN, revista científica, n. 5, 23-43. Recuperado de http://www.ugr.es/ ctriguer/Secundarias/ Master/Curriculum/Bases\%20del\%20ApS.pdf.

Vasen, F. (2013) Las políticas científicas de las universidades Nacionales argentinas en el sistema científico nacional. Ciencia, Docencia y Tecnología, vol. 46(24), 9-32. Recuperado de http://www.redalyc.org/articulo.oa?id=14527692001. 